10,12

\title{
Нелокальные солитоны в нелинейной цепочке атомов
}

\author{
() Т.А. Гаджимурадов, А.М. Агаларов
}

Институт фозики ДФИЦ РАН,

Махачкала, Россия

E-mail: gta-1987@mail.ru

Поступила в Редакцию 30 декабря 2019 г.

В окончательной редакции 30 декабря 2019 г.

Принята к публикации 10 января 2020 г.

Рассмотрена нелинейная 1D-цепочка с нелокальным взаимодействием. Методом многомасштабных разложений получено нелокальное уравнение, описывающее распространение волн огибающих в среде. Изучены свойства полученного уравнения и методом Дарбу преобразований построены точные солитоноподобные решения.

Ключевые слова: $\mathscr{P} \mathscr{T}$-симметрия, солитон, Дарбу преобразование.

DOI: 10.21883 /FTT.2020.06.49341.16M

\section{1. Введение}

После пионерской работы [1] большое внимание в нелинейной теории уделяется интегрируемым нелокальным моделям [2-11]. Этот интерес возник из линейной теории [12], где было показано, что гамильтонианы, коммутирующие с композицией операторов пространственного отражения $(\mathscr{P})$ и инверсии по времени $(\mathscr{T})$ могут обладать действительным спектром. Т. е. эрмитовость не является, необходимым условием вещественнозначности

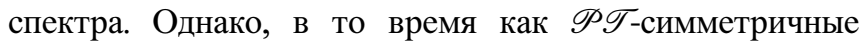
локальные модели нашли широкое применение в различных областях физики [13-25], их нелинейные и одновременно нелокальные аналоги испытывают сложности в физической интерпретации. Это вызвано тем, что в рамках указанных нелокальных уравнений динамика рассматриваемой физической величины $R$ в точке $P\left(\mathbf{r}_{0}, t_{0}\right)$ зависит от значения $R$ в пространственно- и/или временно-зеркальной точке $P_{\mathrm{mir}}=P\left( \pm \mathbf{r}_{0}, \pm t_{0}\right)$. Нарушение принципа локальности придает качественно иные свойства нелокальным нелинейным моделям, отсутствующие в классических моделях, в которых динамика $R$ определяется самой окрестностью точки $P$. Некоторые физические аспекты таких уравнений представлены в работах [26-28].

С другой стороны, большинство классических интегрируемых нелинейных уравнений могут быть рассмотрены как некоторое приближение при описании динамики колебаний цепочки частиц (атомов) при наличии нелинейного взаимодействия. Например, нелинейные волны акустического типа в таких моделях описываются в рамках уравнений Буссинеска или Кортевега-де Фриза и их модификаций [29-31]. Волны же оптического типа - нелинейным уравнением уравнением КлейнаГордона, где нелинейность определяется потенциалом взаимодействия. С учетом выше сказанного, для построения нелокальной математической модели, в данной работе рассматривается классическая моноатомная цепочка с нелокальным взаимодействием. Эта простая механи- ческая модель вносит некоторую ясность в понимании физики нелокального взаимодействия. Как известно, в таких моделях существуют состояния, которые обладают быстрыми внутренними колебаниями. К ним также относятся солитоны огибающей. Динамические свойства их сильно зависят от потенциала взаимодействия. Рассматривая континуальный предел данной модели, методом многих масштабов получено нелокальное нелинейное уравнение, описывающее эволюцию огибающей. Данное уравнение допускает широкий класс солитоноподобных решений. Они представляют собой суперпозицию классических гармонических и нелинейных волн, что крайне необычно для нелинейных локальных моделей.

\section{2. Гамильтониан}

Простая модель, описывающая колебания в цепочке однотипных частиц, взаимодействующих с ближайшими соседями, в присутствии зеркально- нелокального взаимодействия задается потенциалом $U=U_{\text {ngh }}+U_{\text {mir }}$. Динамика продольных колебаний в такой модели описывается стандартным гамильтонианом

$$
\begin{aligned}
H & =K+U \\
& =\sum_{n}\left[\frac{m \dot{q}_{n}^{2}}{2}+U_{\mathrm{ngh}}\left(q_{n+1}-q_{n}\right)+\frac{1}{2} U_{\mathrm{mir}}\left(q_{n}-q_{-n}\right)\right],
\end{aligned}
$$

где $m, q_{n}$ - масса и координата $n$-ой частицы. Пусть $d$ - межатомное расстояние в состоянии равновесия. Будем рассматривать соизмеримое расположение частиц, т.е. стационарное состояние будет соответствовать абсолютному минимуму потенциальной энергии. Потенциал $U_{\mathrm{mir}}(r)$ при этом периодический с периодом $d$. Возможная реализация такой цепочки представлена на рис. 1. Важно отметить, что $U_{\text {mir }}$ не является внешним потенциалом, как например, в модели ФренкеляКанторова [31]. В предположении, что смешение $u_{n}$ 


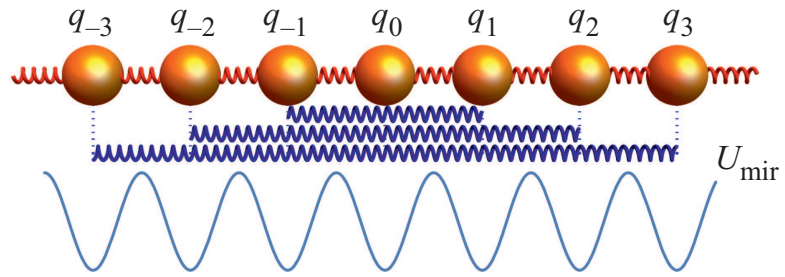

Рис. 1. Схематический рисунок цепочки частиц (атомов), взаимодействующих через два типа пружин. Каждая частица гармонически связана с ближайшими соседями: $F_{\text {ngh }}^{i, i+1} \sim \Delta_{i, i+1}$ и ангармонически с зеркальной частицей $F_{\text {mir }}^{i,-i} \sim \Delta_{i,-i} \pm \Delta_{i,-i}^{3}$, где $\Delta_{i, j}=q_{i}-q_{j}$.
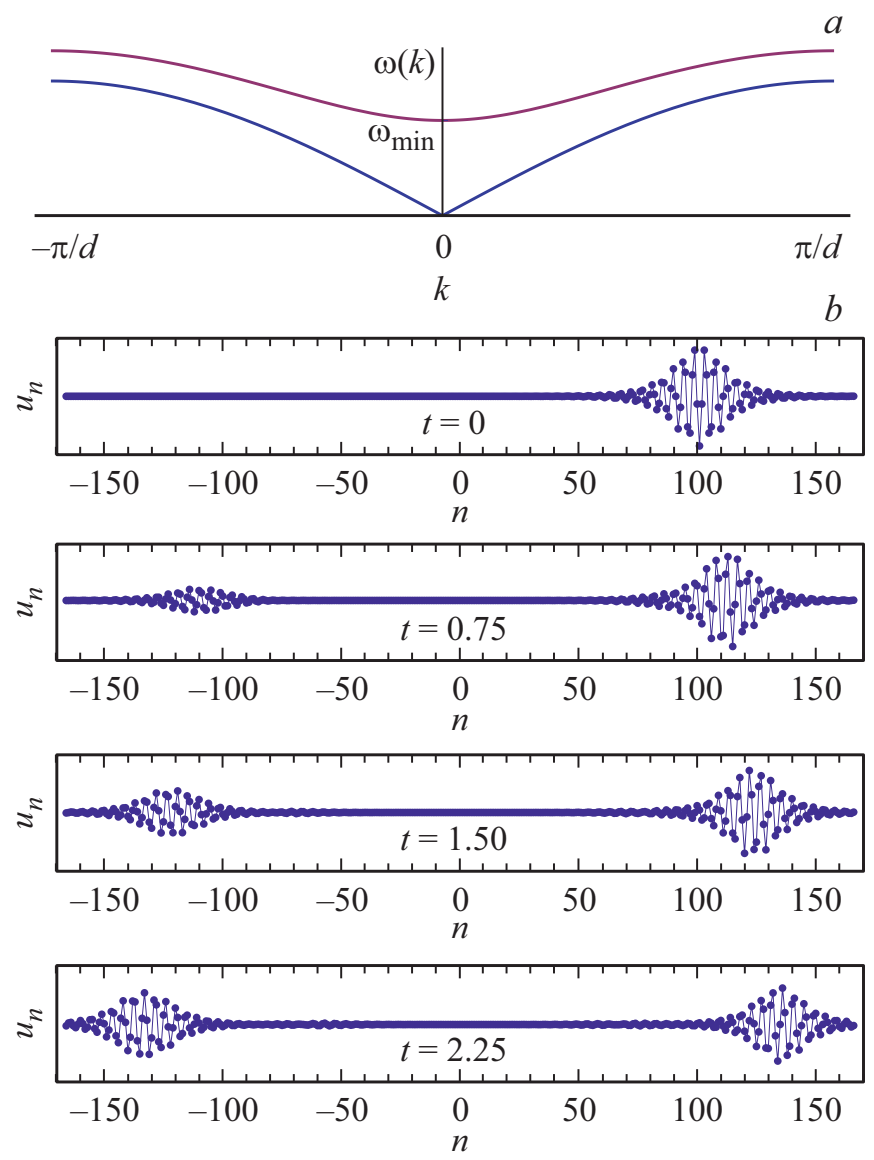

Рис. 2. (a) Распространение локализованного начального возмущения в модели (3). Для наглядности отображено поперечное отклонение $u_{n}$. (b) Акустическая и оптическая ветви дисперсионного соотношения. Значение $w_{\min }=\sqrt{2 \beta^{2}}$ соответствует минимальной оптической частоте.

мало по сравнению с расстоянием между частицами потенциальная энергия системы представима как

$$
\begin{aligned}
U= & \sum_{n}\left[\frac{U_{\text {ngh }}^{\prime \prime}(d)}{2}\left(u_{n+1}-u_{n}\right)^{2}+\frac{U_{\text {mir }}^{\prime \prime}(2 n d)}{4}\left(u_{n}-u_{-n}\right)^{2}\right. \\
& \left.+\frac{U_{\text {mir }}^{(4)}(2 n d)}{48}\left(u_{n}-u_{-n}\right)^{4}\right]
\end{aligned}
$$

Здесь учтено для $U_{\mathrm{ngh}}(r)$ гармоническое приближение, а для $U_{\mathrm{mir}}(r)$ нелинейность четвертого порядка. Уравнение движения дискретной цепи принимает вид

$$
\begin{aligned}
\ddot{u}_{n}= & \alpha^{2}\left(u_{n-1}-2 u_{n}+u_{n+1}\right) \\
& -\beta^{2}\left(u_{n}-u_{-n}\right)+\gamma\left(u_{n}-u_{-n}\right)^{3},
\end{aligned}
$$

где $\alpha^{2}=U_{\text {ngh }}^{\prime \prime}(d) / m, \beta^{2}=U_{\text {mir }}^{\prime \prime}(d) / m$ и $\gamma=-U_{\text {mir }}^{(4)}(d) / 6 m$. Последние два слагаемых в данном уравнении учитывают нелокальное взаимодействие. В общем случае потенциала коэффициент ангармонизма $\gamma$ может быть как отрицательным, так и положительным. Очевидно, что любое возмушение для $u_{n}$ распространиться на $u_{n \pm 1}$, и что более важно на $u_{-n}$. Для наглядности на рис. 2, $а$ приведены результаты численного моделирования динамики начального возмущения локализованного в окрестности узла $n=100$.

Линейный случай. При $\gamma=0$ предложенная модель является линейной. Очевидно, что при этом уравнение (3) допускает только стоячие волны

$$
\begin{gathered}
u_{n}=A e^{i\left(k n d-w_{ \pm} t\right)} \pm A e^{i\left(-k n d-w_{ \pm} t\right)}+\text { c. c. } \\
w_{+}^{2}=4 \alpha^{2} \sin ^{2} \frac{k d}{2}, \quad w_{-}^{2}=2 \beta^{2}+w_{+}^{2}
\end{gathered}
$$

Т.е. уравнение (3) допускает два дисперсионных соотношения, не смотря на то, что цепочка является моноатомной. Ветви колебаний представлены на рис. 2, $b$. Наличие двух ветвей обусловлено наличием двух видов взаимодействия между частицами. Ветвь $w=w_{+}$ соответствует акустическому типу колебаний $-u_{n}$ и $u_{-n}$ колеблются в фазе, а ветвь $w=w_{-}$является оптической - колебания зеркально симметричных частиц происходят в противофазе.

\section{3. Уравнение волны огибающей}

Рассмотрим распространение нелинейных волн огибающей в данной модели. Наиболее просто описать эти нелинейные моды в континуальном приближении цепочки. Для этого воспользуемся непрерывной моделью для (3). Предполагая $x=n d$ и переходя к пределу $d \rightarrow 0$ имеем непрерывный аналог уравнения (3)

$$
\frac{\partial^{2} u(x, t)}{\partial t^{2}}=\tilde{\alpha}^{2} \frac{\partial^{2} u(x, t)}{\partial x^{2}}-\beta^{2} \Delta+\gamma \Delta^{3},
$$

где $\Delta=u(x, t)-u(-x, t)$ и $\tilde{\alpha}=d \alpha$.

Как видно, приведенное выше уравнение не учитывает эффекты, обусловленные дискретностью цепочки [32].

Для описания динамики огибающей волновых пакетов в рамках (5) воспользуемся методом многих масштабов. Представим решение в виде ряда $u=\varepsilon u_{1}+\varepsilon^{2} u_{2}+\varepsilon^{3} u_{3}+\cdots$, причем $u_{i}$ являются функциями двух пространственных $x_{i}=\varepsilon^{i} x, i=0,1$, и трех 
$t_{s}=\varepsilon^{s} t, s=\overline{0,2}$ временных переменных. Выделив члены одинаковых порядков малости по $\varepsilon$ в первом порядке получим

$$
\widehat{L} u_{1}=0, \quad \widehat{L}=\frac{\partial^{2}}{\partial t_{0}^{2}}-\tilde{\alpha}^{2} \frac{\partial^{2}}{\partial x_{0}^{2}}+\beta^{2}(1-\mathscr{P}),
$$

где $\widehat{L}$ - оператор, соответствующий линейной части (5). Решение уравнения (6) выберем в виде квазигармонической волны с амплитудой, зависящей от медленных переменных

$$
u_{1}=A\left(x_{1}, t_{1}, t_{2}\right)\left[e^{i \theta}-e^{i \theta^{\mathscr{P}}}\right]+\text { c. c. }
$$

где $f^{\mathscr{P}}(x, t)=f(-x, t)$-инверсия всех пространственных переменных функции $f$. Так как в (5) возможны только стоячие волны, рассматривается именно модуляция стоячих волн. Благодаря тому, что (5) как и (3) допускает два дисперсионных соотношения,

$$
\omega_{1}^{2}=\tilde{\alpha}^{2} k^{2}, \quad \omega_{2}^{2}=2 \beta^{2}+\tilde{\alpha}^{2} k^{2},
$$

высокочастотную составляющую можно выбрать двумя способами $\theta\left(x_{0}, t_{0}\right)=k x_{0}-\omega_{j} t_{0}$, где $\omega_{j} \quad$ и $k$ связаны дисперсионными соотношениями (8). Однако при $\omega=\omega_{1}$ рассматривая цепочка в континуальном пределе представляет недиспергирующую среду. Поэтому далее рассмотрим только случай $\omega=\omega_{2}$.

Члены порядка $\varepsilon^{2}$ приводят к уравнению

$$
\begin{aligned}
\widehat{L} u_{2}= & 2 \tilde{\alpha}^{2} \frac{\partial u_{1}}{\partial x_{0} \partial x_{1}}-2 \frac{\partial u_{1}}{\partial t_{0} \partial t_{1}}=2 i\left(\omega \frac{\partial A}{\partial t_{1}}+\tilde{\alpha}^{2} k \frac{\partial A}{\partial x_{1}}\right) e^{i \theta} \\
& +2 i\left(-\omega \frac{\partial A}{\partial t_{1}}+\tilde{\alpha}^{2} k \frac{\partial A}{\partial x_{1}}\right) e^{i \theta^{\mathscr{P}}}+\text { c. c. }
\end{aligned}
$$

Поскольку действие оператора $\widehat{L}$ на функцию $e^{i \theta}-e^{i \theta^{\mathscr{P}}}$ дает нуль, решение уравнения (9) носит секулярный характер. Способ избежать этого состоит в том, чтобы приравнять к нулю члены, стоящие в правой части. Объединяя эти условия очевидно получим

$$
\frac{\partial^{2} A}{\partial t_{1}^{2}}-v_{g}^{2} \frac{\partial^{2} A}{\partial x_{1}^{2}}=0
$$

где групповая скорость определена как $v_{g}=d w / d k$. Таким образом, в первом приближении возмущения огибающей распространяются с групповой скоростью. При этом имеются две характеристики. Графически это продемонстрировано на рис. 2. Теперь (9) принимает вид $\widehat{L} u_{2}=0$, следовательно, можно положить $u_{2}=0$.

И наконец для членов порядка $\varepsilon^{3}$ получаем

$$
\begin{aligned}
& \widehat{L} u_{3}=\left(\frac{\partial^{2} A}{\partial t_{1}^{2}}-2 i \omega \frac{\partial A}{\partial t_{2}}-\tilde{\alpha}^{2} \frac{\partial A^{2}}{\partial x_{1}^{2}}\right. \\
& \left.+9 \gamma\left(A+A^{\mathscr{P}}\right)\left|A+A^{\mathscr{P}}\right|^{2}\right)\left[e^{i \theta}-e^{i \theta^{\mathscr{P}}}\right]+\Xi+\text { c. c. }
\end{aligned}
$$

где $\Xi$ содержит только гармоники высших порядков. Из-за громоздкости явное выражение для $\Xi$ не приведено. Во избежание секулярности положим коэффициенты равным нулю. С учетом (10), получим

$$
i \frac{\partial A}{\partial t_{2}}+\frac{\omega^{\prime \prime}}{2} \frac{\partial^{2} A}{\partial x_{1}^{2}}+\frac{9 \gamma}{2 \omega}\left(A+A^{\mathscr{P}}\right)\left|A+A^{\mathscr{P}}\right|^{2}=0,
$$

где $\omega^{\prime \prime}=d^{2} \omega / d k^{2}=2 \tilde{\alpha}^{2} \beta^{2} / \omega^{3}$. Данное уравнение описывает динамику комплексной огибающей в рамках модели (3). Заметим, что нелинейный член одновременно является и нелокальным.

\section{4. Солитонные решения}

Уравнение (12) можно записать в новый переменных $t_{2} \rightarrow \tau, x_{1} \rightarrow \sqrt{\omega^{\prime \prime} / 2} \xi$ и $A \rightarrow \sqrt{2 \omega / 9|\gamma|} \psi$, что дает

$$
i \frac{\partial \psi}{\partial \tau}+\frac{\partial^{2} \psi}{\partial \xi^{2}}+\sigma\left(\psi+\psi^{\mathscr{P}}\right)\left|\psi+\psi^{\mathscr{P}}\right|^{2}=0
$$

где $\sigma= \pm 1$ и $\psi^{\mathscr{P}}=\psi(-\xi, \tau)$. Данное уравнение допускает представление в виде системы нелинейного и линейного уравнений Шрёдингера для свободной частицы

$$
\begin{gathered}
i \partial_{\tau} s+\partial_{\xi \xi} s+2 \sigma s|s|^{2}=0, \\
i \partial_{\tau} r+\partial_{\xi \xi} r=0,
\end{gathered}
$$

где $s=\psi+\psi^{\mathscr{P}}$ и $r=\psi-\psi^{\mathscr{P}}$. Далее, в рамках данной статьи ограничимся случаем $\sigma=1$. Решение линейного уравнения (14b) представим в виде

$$
r=2 I(\xi, \tau)=\int_{\mathbb{R}} \rho(\mu) \sin (\mu \xi) e^{-i \mu^{2} \tau} d \mu,
$$

где $\rho$ произвольная интегрируемая функция.

Известно, что нелинейное уравнение Шрёдингера вложимо в спектральную задачу Захарова-Шабата

$$
\begin{gathered}
\partial_{\xi} \Psi=J \Psi \Lambda+Q \Psi \\
\partial_{\tau} \Psi=2 J \Psi \Lambda^{2}+2 Q \Psi \Lambda+\left(J Q^{2}-J \partial_{\xi} Q\right) \Psi, \\
Q=\left(\begin{array}{cc}
0 & i \bar{s} \\
i s & 0
\end{array}\right), \quad J=\left(\begin{array}{cc}
i & 0 \\
0 & -i
\end{array}\right), \quad \Lambda=\left(\begin{array}{ll}
\lambda & 0 \\
0 & \bar{\lambda}
\end{array}\right)
\end{gathered}
$$

и решения могут быть получены методом Дарбу преобразований [33]. Если для выбранного затравочного решения $s_{[0]}$ имеются $N$ собственных функций $\Psi_{l}$

$$
\Psi_{i}=\left(\begin{array}{ll}
\phi_{2 l-1} & \phi_{2 l} \\
\varphi_{2 l-1} & \varphi_{2 l}
\end{array}\right), \quad \Lambda_{l}=\left(\begin{array}{cc}
\lambda_{2 l-1} & 0 \\
0 & \lambda_{2 l}
\end{array}\right), \quad l=\overline{1, N}
$$

где $\lambda_{2 l}=\bar{\lambda}_{2 l-1}, \varphi_{2 l-1}=-\bar{\phi}_{2 l}, \varphi_{2 l}=\bar{\phi}_{2 l-1}$, то результат после $N$-ой итерации дается как

$$
s_{[N]}=s_{[0]}-2 \frac{\Delta_{1}}{\Delta_{2}}
$$


где

$$
\begin{aligned}
\Delta_{1} & =\left|\begin{array}{cccc}
\lambda_{1}^{N} \varphi_{1} & \lambda_{2}^{N} \varphi_{2} & \ldots & \lambda_{2 N}^{N} \varphi_{2 N} \\
\lambda_{1}^{N-1} \varphi_{1} & \lambda_{2}^{N-1} \varphi_{2} & \ldots & \lambda_{2 N}^{N-1} \varphi_{2 N} \\
\ldots & \ldots & \ldots & \ldots \\
\varphi_{1} & \varphi_{2} & \ldots & \varphi_{2 N} \\
\lambda_{1}^{N-2} \phi_{1} & \lambda_{2}^{N-2} \phi_{2} & \ldots & \lambda_{2 N}^{N-2} \phi_{2 N} \\
\ldots & \ldots & \ldots & \ldots \\
\phi_{1} & \phi_{2} & \ldots & \phi_{2 N}
\end{array}\right|, \\
\Delta_{2} & =\left|\begin{array}{cccc}
\lambda_{1}^{N-1} \phi_{1} & \lambda_{2}^{N-1} \phi_{2} & \ldots & \lambda_{2 N}^{N-1} \phi_{2 N} \\
\ldots & \ldots & \ldots & \ldots \\
\phi_{1} & \phi_{2} & \ldots & \phi_{2 N} \\
\lambda_{1}^{N-1} \varphi_{1} & \lambda_{2}^{N-1} \varphi_{2} & \ldots & \lambda_{2 N}^{N-1} \varphi_{2 N} \\
\ldots & \ldots & \ldots & \ldots \\
\varphi_{1} & \varphi_{2} & \ldots & \varphi_{2 N}
\end{array}\right|,
\end{aligned}
$$

Однако в данном случае необходимо $s=s^{\mathscr{P}}$, что накладывает некоторые ограничения.

Светлые солитоны: случай нулевых граничных условий для $s(\xi, \tau)$. При $s_{[0]}=0$ из уравнений $(16),(17)$ элементы собственной матрицы $\Psi_{j}$ могут быть построены с использованием

$$
\phi_{j}=A_{j} e^{i \lambda_{j} \xi+2 i \lambda_{j}^{2}\left(\tau-\tau_{0}\right)}, \quad j=\overline{1,2 N}
$$

Пусть имеется набор амплитуд и собственных значений

$$
\begin{aligned}
\mathscr{A} & =\left\{A_{1}, A_{2}, \ldots, A_{2 m-1}, A_{2 m}, A_{2 m+1}, \ldots, A_{2 N}\right\}, \\
\mathfrak{L} & =\left\{\lambda_{1}, \lambda_{2}, \ldots, \lambda_{2 m-1}, \lambda_{2 m}, \lambda_{2 m+1}, \ldots, \lambda_{2 N}\right\},
\end{aligned}
$$

где $m$ четно (может равняться $N$ ). Тогда достаточным условием четности $s$ будет являться

$$
\begin{aligned}
\text { (I) } \lambda_{(2 p+1) R} & =-\lambda_{(2 p-1) R}, \quad \lambda_{(2 p+1) I}= \pm \lambda_{(2 p-1) I}, \\
A_{2 p} & =\operatorname{sign}\left(\lambda_{(2 p-1) I}\right) \bar{A}_{2 p-1}, p=\overline{1, m-1} ; \\
\text { (II) } \lambda_{(2 q+1) R} & =0, \quad\left|A_{2 q+2}\right|=\left|A_{2 q+1}\right|, \quad q=\overline{m, N-1},
\end{aligned}
$$

где индексы $R, I$ соответствуют действительной и мнимой частям. При этом собственные значения $\lambda_{1}, \ldots, \lambda_{2 m}$ соответствуют зеркально симметрично распространяющимся солитонам, а $\lambda_{2 m+1}, \ldots, \lambda_{2 N}-$ связанным, периодически сталкивающимся состояниям. Параметр $\tau_{0}$ соответствует моменту времени их взаимодействия. И наконец $\psi(\xi, \tau)$ может быть представлено как

$$
\psi(\xi, \tau)=I(\xi, \tau)-\frac{\Delta_{1}}{\Delta_{2}}
$$

Например односолитонное решение при $\rho(\eta)=\delta(\eta-1)$, где $\delta$ функция Дирака может быть представлено как

$$
\psi(\xi, \tau)=\sin (\xi) e^{-i \tau}+B \operatorname{sech}\left(2 \lambda_{I} \xi\right) e^{4 i \lambda_{I}^{2}\left(\tau-\tau_{0}\right)},
$$

где $B=i \lambda_{1} \bar{A}_{1} / A_{2}$. Таким образом, уравнение (13) допускает как солитонные решения, так и не распадающиеся

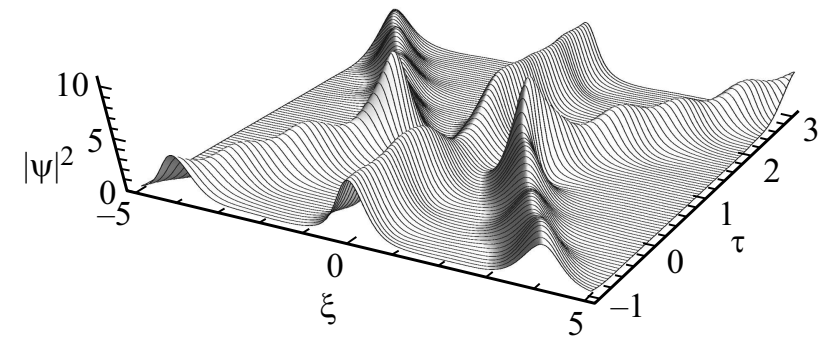

Рис. 3. Динамика трехсолитонного решения уравнения (13) при $\rho(\eta)=0.2 \delta(\eta-2)$. Параметры выбраны согласно (I) и (II): $A_{1}=e^{2 i}, A_{2}=-e^{-2 i}, A_{3}=3 e^{i}, A_{4}=3 e^{-i}, A_{5}=2 e^{4 i}$, $A_{6}=-2 e^{3 i}, \lambda_{1}=-0.4-i, \lambda_{3}=0.4+i, \lambda_{5}=1.2 i$.

волновые пакеты. Это вызвано тем, что в исходной решеточной модели присутствуют два типа связей: линейная и нелинейная. Для наглядности на рис. 3. представлено трехсолитонное решение, однако явное выражение для краткости опущено.

Бризеры: ненулевые граничные условия для $s(\xi, \tau)$. Более интересные состояния могут быть получены при периодическом затравочном решении. Преследуя цель получения пространственно-четного решения, затравочное решение выберем виде

$$
s_{[0]}=A e^{2 i A^{2}\left(\tau-\tau_{0}\right)},
$$

Без ограничения общности можно можно $A$ действительным. Соответствующие $\phi_{1}$ и $\varphi_{1}$ могут быть найдены из (16). Рассмотрим три случая для корней характеристического уравнения $\lambda^{2}+A^{2}+\mu^{2}=0$. Отметим, что если $\mu$ является корнем, то таковым являться и $-\mu$

Солитон Перегрина. Если корни характеристического уравнения равны нулю $\mu=0$, т. е. $\lambda_{1}= \pm i A$, то

$$
\begin{gathered}
\phi_{1}=\left[\xi+2\left(\tau-\tau_{0}\right) \lambda_{1}-\frac{i}{2 \lambda_{1}}\right] e^{i A^{2}\left(\tau-\tau_{0}\right)} \\
\varphi_{1}=\frac{1}{2 A}\left[-2 \lambda_{1} \xi+4 A^{2}\left(\tau-\tau_{0}\right)-i\right] e^{-i A^{2}\left(\tau-\tau_{0}\right)}
\end{gathered}
$$

Отсюда

$$
\psi=I(\xi, \tau)+\left[-\frac{1}{2}+\frac{2+8 i A^{2}\left(\tau-\tau_{0}\right)}{4 A^{2} \xi^{2}+16 A^{4}\left(\tau-\tau_{0}\right)^{2}+1}\right] s_{[0]},
$$

На рис. 4, $a$ представлено данное решение. Пик плотности относительно осциллирующего фона локализован как в пространстве (в окрестности $\xi=0$ ) так и во времени (в окрестности $\tau=\tau_{0}$ ). Такого рода решения как известно, описывают „волны-убийцы“ в нелинейной теории [34-36].

Ахмедиева бризер. В случае $\mu \neq 0$, собственные функции могут быть представлены как

$$
\begin{aligned}
& \phi_{1}=\left[e^{\varrho}+\frac{i \lambda_{1}-\mu}{A} e^{-\varrho}\right] e^{-i A^{2}\left(\tau-\tau_{0}\right)}, \\
& \varphi_{1}=\left[i e^{-\varrho}-\frac{\lambda_{1}+i \mu}{A} e^{\varrho}\right] e^{i A^{2}\left(\tau-\tau_{0}\right)},
\end{aligned}
$$

где введено обозначение $\varrho=2 \mu \xi+4 \lambda_{1} \mu\left(\tau-\tau_{0}\right)$. 


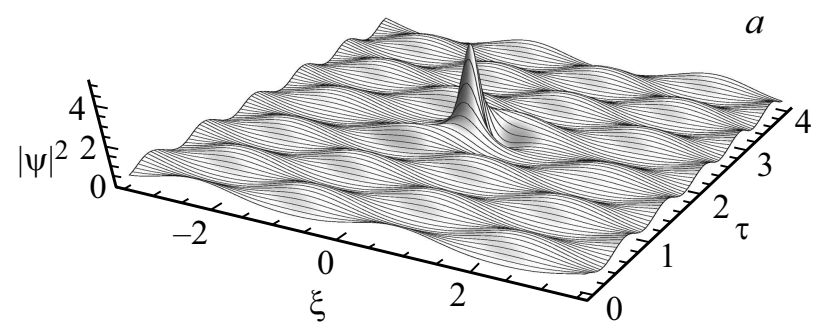

$b$

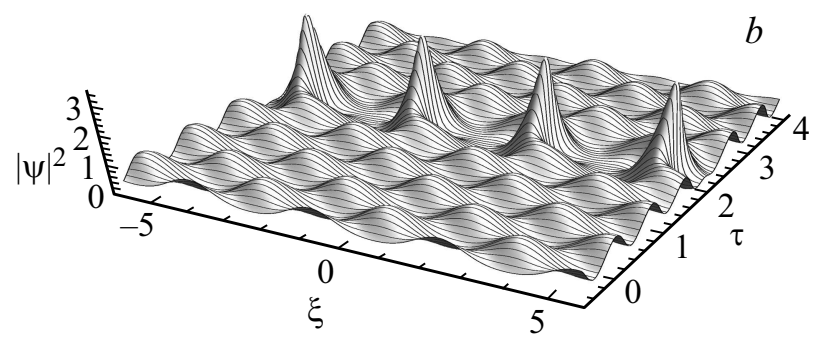

C

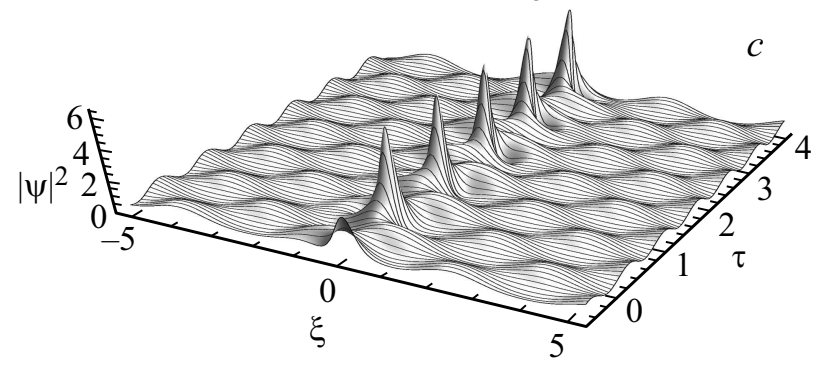

Рис. 4. Бризерные состояния уравнения (13) при $\rho(\eta)=0.6 \delta(\eta-2), A=1.5$ и $\tau_{0}=2$. (a) Солитон Перегрина: $\mu=0 . \quad(b)$ Ахмедиева бризер при $\mu=i$ и $\lambda_{1}=\sqrt{5} i / 2$. (c) Солитон Кузнецова-Ма при $\mu=1$ и $\lambda_{1}=\sqrt{13} i / 2$.

Ахмедиева бризер соответствует случаю $\mu=i \mu_{I}$ и решение может быть представлено как

$$
\psi=I(\xi, \tau)+\left[-\frac{1}{2}+\frac{\mu_{I} \cosh \vartheta+i \lambda_{1} A \sinh \vartheta}{A \cosh \vartheta-\lambda_{1 I} \cos \left(2 \xi \mu_{I}\right)} \frac{\mu_{I}}{A}\right] s_{[0]}
$$

где $\vartheta=4 \mu_{I} \lambda_{1 I}\left(\tau-\tau_{0}\right)$. Отметим, что при $A^{2} \leq \mu_{I}^{2}$ решение вырождается в плоскую волну. Данное решение отображено на рис. $4, b$ и является локализованным во времени в окрестности $\tau=\tau_{0}$.

Солитон Кузнецова-Ма. Данное решение соответствует случаю $\mu=\mu_{R}$. Решение может быть представлено как

$$
\psi=I(\xi, \tau)+\left[-\frac{1}{2}+\frac{\mu_{R} \cos \vartheta+i \lambda_{1 I} A \sin \vartheta}{A \cos \vartheta-\lambda_{1 I} \cosh \left(2 \xi \mu_{R}\right)} \frac{\mu_{R}}{A}\right] s_{[0]},
$$

где $\vartheta=4 \mu_{R} \lambda_{1 I}\left(\tau-\tau_{0}\right)$. Оно представляет локализованное в пространстве состояние (см. рис. $4, c$ ).

Стоит отметить, что (13) допускает бризерные решения более высоких порядков [34-39]. Общего решения бегущего бризера модель не допускает, так как не обладает трансляционной симметрией, что в свою очередь вызвано симметрией уравнения (13) относительно пространственного отражения $\xi \rightarrow-\xi$. Легко также заметить, что бризерные решения обладают осциллирующей фоновой плотностью, что связано с интерференцией $s_{[0]}(\tau)$ и $I(\xi, \tau)$. Такого рода картина для фоновой плотности обнаруживается и в ряде других моделей [40-43].

\section{5. Заключение}

Таким образом, рассмотрев цепочку с необычной нелокальной связью между зеркально симметричными частицами, получен новый тип нелокального нелинейного уравнения. Решения полученного уравнения представимы в виде $\psi=$,linear wave“ + ,soliton“, т. е. представляют собой суперпозицию линейных и нелинейных волн. При этом линейная волна должна быть пространственно-нечетной, а солитонная часть пространственночетной. Т.е. двигающиеся солитоны могут возникать в такой среде только парами, что присуще многим нелокальным моделям $[1,6]$. Такого рода суперпозиции не могут возникать в классических локальных нелинейных моделях.

Стоит отметить, что такое взаимодействие между частицами, которые не находятся в непосредственной окрестности, не является математической абстракцией. Не сложно сопоставить исходной механической модели физически реализуемую нелинейную электрическую цепь. Нелокальное взаимодействие здесь может быть достигнуто с помощью „нелокальных“ нелинейных элементов, соединяющие не соседние узлы цепочки. Исследования распространения сигнала в аналогичных локальных цепочках являлись одними из первых, которые позволили экспериментально наблюдать солитоны и проверить фундаментальные свойства солитонов [44-46].

В настоящей работе мы ограничились только „фокусирующим“ случаем $\sigma=1$. Построение точных солитонных решений при дефокусирующей нелинейности $\sigma=-1$ также представляет интерес. В данном случае, солитонная часть представляется темными солитонами. Более того интересен случай когда, взаимодействие между ближайшими соседями носит ангармонический характер. Эти и другие вопросы будут рассмотрены в следующей работе.

\section{Финансирование работы}

Работа выполнена в рамках госзадания Института физики ДФИЦ РАН.

\section{Конфликт интересов}

Авторы заявляют, что у них нет конфликта интересов.

\section{Список литературы}

[1] M.J. Ablowitz, Z.H. Musslimani. Phys. Rev. Lett. 110, 064105 (2013).

[2] M.J. Ablowitz, Z.H. Musslimani. Stud. Appl. Math. 139, 7 (2017).

[3] D. Sinha, P.K. Ghosh. Phys. Rev. E 91, 042908 (2015). 
[4] M.J. Ablowitz, Z.H. Musslimani. Phys. Rev. E 90, 032912 (2014).

[5] M. Li, T. Xu. Phys. Rev. E 91, 033202 (2015).

[6] M.J. Ablowitz, Z.H. Musslimani. Nonlinearity 29, 915 (2016).

[7] A.S. Fokas. Nonlinearity 29, 319 (2016).

[8] J.-L. Ji, Z.-N. Zhu. Commun. Nonlinear Sci. Numer. Simul. 42, 699 (2017).

[9] B. Yang, J. Yang. Stud. Appl. Math. 140, 178 (2018).

[10] V.S. Gerdjikov, A. Saxena. J. Math. Phys. 58, 013502 (2017).

[11] S. Stalin, M. Senthilvelan, M. Lakshmanan. Phys. Lett. A 381, 2380 (2017).

[12] C.M. Bender, S. Boettcher. Phys. Rev. Lett. 80, 5243 (1998).

[13] C.E. Rüter, K.G. Makris, R. El-Ganainy, D.N. Christodoulides, M. Segev, D. Kip. Nature Phys. 6, 192 (2010).

[14] R. El-Ganainy, K.G. Makris, D.N. Christodoulides, Z.H. Musslimani. Opt. Lett. 32, 2632 (2007).

[15] A. Regensburger, C. Bersch, M.-A. Miri, G. Onishchukov, D.N. Christodoulides, U. Peschel. Nature 488, 167 (2012).

[16] K.G. Makris, R. El-Ganainy, D.N. Christodoulides, Z.H. Musslimani. Phys. Rev. Lett. 100, 103904 (2008).

[17] C.M. Bender, D.C. Brody, H.F. Jones. Phys. Rev. Lett. 89, 270401 (2002).

[18] C.M. Bender. Rep. Prog. Phys. 70, 947 (2007).

[19] G.L. Giorgi. Phys. Rev. B 82, 052404 (2010).

[20] J.M. Lee, T. Kottos, B. Shapiro. Phys. Rev. B 91, 094416 (2015).

[21] C.M. Bender, S.P. Klevansky. Phys. Rev. Lett. 105, 031601 (2010).

[22] C.M. Bender, D.C. Brody, H.F. Jones. Phys. Rev. Lett. 93, 251601 (2004).

[23] A. de Souza Dutra, V.G.C.S. dos Santos, A.C. Amaro de Faria. Phys. Rev. D 75, 125001 (2007).

[24] J. Schindler, A. Li, M.C. Zheng, F.M. Ellis, T. Kottos. Phys. Rev. A 84, 040101 (2011).

[25] Z. Lin, J. Schindler, F.M. Ellis, T. Kottos. Phys. Rev. A 85, 050101 (2012).

[26] T.A. Gadzhimuradov, A.M. Agalarov. Phys. Rev. A 93, 062124 (2016).

[27] S. Lou, F. Huang. Sci. Rep. 7, 869 (2017).

[28] X. yan Tang, Z. feng Liang, X. zhi Hao. Commun. Nonlinear Sci. Numer. Simul. 60, 62 (2018).

[29] M. Toda. Theory of nonlinear lattices. Springer Science \& Business Media (2012). V. 20..

[30] A.M. Kosevich, A.S. Kovalev. An introduction to nonlinear physical mechanics. Nauk. Dumka, Kiev (1989).

[31] O.M. Braun, Y.S. Kivshar. The Frenkel-Kontorova model: concepts, methods, and applications. Springer Science \& Business Media (2013).

[32] P. Rosenau. Phys. Lett. A 118, 222 (1986).

[33] V. Matveev, M. Salle. Darboux Transformations and Soliton. Springer-Verlag, Berlin (1991).

[34] N. Akhmediev, A. Ankiewicz, M. Taki. Phys. Lett. A 373, 675 (2009).

[35] F. Baronio, A. Degasperis, M. Conforti, S. Wabnitz. Phys. Rev. Lett. 109, 044102 (2012).

[36] A.A. Gelash. Phys. Rev. E 97, 022208 (2018).

[37] D.J. Kedziora, A. Ankiewicz, N. Akhmediev. Phys. Rev. E 84, 056611 (2011).
[38] P. Dubard, V.B. Matveev. Nature Haz. Earth Syst. Sci. 11, 667 (2011).

[39] D.J. Kedziora, A. Ankiewicz, N. Akhmediev. Phys. Rev. E 85, 066601 (2012).

[40] T.A. Gadzhimuradov, G.O. Abdullaev, A.M. Agalarov. Nonlinear Dynamics 89, 2695 (2017).

[41] Y.-Q. Yuan, B. Tian, L. Liu, Y. Sun, Z. Du. Chaos, Solitons \& Fractals 107, 216 (2018).

[42] A.M. Agalarov, V.G. Zhulego, T.A. Gadzhimuradov. Phys. Rev. E 91, 042909 (2015).

[43] H.J. Shin, J. Phys. A 37, 8017 (2004).

[44] R. Hirota, K. Suzuki. Proc. IEEE 61, 1483 (1973).

[45] P. Marqui/‘e, J.M. Bilbault, M. Remoissenet. Phys. Rev. E 51, 6127 (1995).

[46] T. Kuusela. Phys. Lett. A 167, 54 (1992).

Редактор Т.Н. Василевская 